\title{
Aluminium-surfaced Mirrors
}

By Dr. H. Spencer Jones, F.r.S.

$\mathrm{T}$ HE mirrors in astronomical reflecting telescopes were formerly made of speculum metal-a hard alloy of copper and tin capable of taking a fine polish and having a fairly high reflecting power. Speculum metal mirrors have been entirely replaced by glass mirrors, coated on the figured surface with a thin film of silver. The silvered surface, when fresh, has a high reflecting power for wave-lengths greater than $0.375 \mu$; at this wave-length the reflecting power is 0.80 ; at $0.400 \mu$ it is 0.85 ; at $0.450 \mu$ it is 0.90 and at $0.700 \mu$ it is 0.95 . On the short wave-length side of $0.375 \mu$ the reflecting power falls off rapidly, owing to selective absorption, to a minimum value at about $0.315 \mu$ of only 0.04 . This is a serious disadvantage when observations are required in the ultra-violet region. Thus, for example, at a wave-length of about $0.325 \mu$ the atmospheric transmission is 0.50 but the reflecting power of silver is only 0.12 . In a reflecting telescope, the image is normally produced by reflection at two silvered mirrors, so that the loss by the selective absorption of the silver is much greater than the above figures indicate.

These figures refer to a freshly deposited silver film. But the reflecting power steadily falls owing to gradual oxidation or to tarnishing due to the action of sulphur dioxide in the atmosphere. Where such atmospheric contamination is prevalent the reflecting power falls rapidly. At even the most favourable sites, however, there is a gradual fall in reflecting power, so that the mirrors must be periodically dismounted and resilvered. The silver film is easily deposited chemically and though the silvering process itself is not difficult, any satisfactory method of making it unnecessary would be welcomed, for it would imply that the decrease in reflecting power-which necessitates lengthened exposures and is particularly troublesome in some photometric work-had been eliminated. Coating the silver film with a thin film of colourless lacquer has been tried, but the optical perfection of figure is almost inevitably impaired.

The development of a method of coating glass surfaces with a film of aluminium and the unexpected properties of such films are, therefore, of the greatest importance for astronomical observation. For wave-lengths greater than about $0.40 \mu$, the reflecting power of an aluminium film is somewhat less than that of a freshly deposited silver film. The difference is not, however, very great; at a wave-length of $0.50 \mu$ the reflecting power of the aluminium film is 0.88 as compared with 0.91 for silver; at $0.60 \mu$, the relative values are 0.89 and 0.93 ; at $0.70 \mu, 0.87$ and 0.95 . The reflecting power of a silver film after a short period of use would soon fall below that of a freshly deposited aluminium film. To the short wave-length side of $0.40 \mu$, the aluminium film is greatly superior to the silver film, as it does not show the band due to selective absorption. At $0.35 \mu$, its reflecting power is 0.85 , as contrasted with 0.70 for silver; at $0.30 \mu$ (near the limit of atmospheric transmission), it is 0.83 as compared with 0.08 for silver. For observations in the ultra-violet region, the aluminium film is, therefore, very much superior to the silver film.

'The aluminium films have other important advantages. A freshly deposited film on exposure to the air immediately oxidises, and the oxide coating forms a protective layer which prevents the film from tarmishing. It is stated by Dr. J. Strong, who has developed at the California Institute of Technology a technique for the coating of mirrors with aluminium, that concentrated nitric acid can be poured on the mirror with immunity. Nevertheless, the film can be readily dissolved by dilute hydrochloric acid to which a trace of some copper salt has been added. Sulphur dioxide in the atmosphere does not tarnish an aluminium film, or at most at a very slow rate. A small mirror partly coated with silver and partly with aluminium has been exposed at Greenwich, where conditions as regards sulphur in the atmo. sphere are bad, until the silver film had become completely yellow. The aluminium film was apparently unaffected. Dr. Strong mentions that the mirrors of a telescope aluminised in October 1932 and constantly used since show as yet no signs of tarnish.

Aluminium films are more strongly adherent to glass than silver films. Dr. Strong states that a piece of adhesive tape may be pressed on to the film and then stripped off without loosening the metal from the glass. This tenacity makes it possible to clean the surface of dust or other contamination by washing with soap and water. It is also stated that aluminised mirrors do not scatter light.

The largest mirror yet coated with aluminium is the 36-inch mirror of the Crossley reflector of the Lick Observatory, which was coated in December 1933. When this mirror was coated with silver, a long exposure spectrogram ended at about $0.325 \mu$. An equal exposure with the aluminised mirror gives a spectrogram extending to about $0 \cdot 300 \mu$. An exposure of only 20 seconds on the star ` Orionis, of magnitude $2 \cdot 9$, gave a spectrogram measurable to about $0 \cdot 310 \mu$. The atmospheric ozone absorption lines in the ultraviolet can be photographed in a few seconds. Dr. W. H. Wright states that comparison of photographs of the north polar sequence, before and after the aluminising, indicated that the general reflectivity had been stepped up by 50 or 60 per cent; this illustrates the effect of tarmishing of a silver film even under the favourable 
atmospheric conditions on the summit of Mount Hamilton.

Aluminium is a difficult metal to sputter by cathode disintegration, and the most effective method of depositing the aluminium films has been found to be by an evaporation process in a high vacuum of the order of $0.0001 \mathrm{~mm}$. of mercury. The aluminium is heated in small tungsten coils arranged opposite the mirror to be coated; the aluminium evaporates and condenses on the face of the mirror. The low pressure enables each atom of aluminium, after evaporation, to travel in a straight path to the mirror with small probability of collision with other atoms. In coating the mirror of the Crossley reflector, twelve helical tungsten coils were arranged round a 36-inch circle at a distance of 18 inches from the mirror. Each coil had 10 turns and to each turn a U-shaped aluminium wire, $\frac{3}{8}$ inch long and $\frac{1}{32}$ inch diameter, was clamped. Each coil was connected in turn to the electrical supply, at a voltage of 20 volts, the entire process of distilling from the twelve coils requiring about three minutes. The mirror was placed on brass bars fastened to a reinforced steel bedplate, one inch in thickness ; this arrangement enabled gases underneath the mirror to be pumped out easily. The bell-jar covering the mirror was of $\frac{1}{4}$-inch sheet steel, stiffened at the bottom by a rolled angle iron, and machined to give a perfect plane surface. A lead fuse wire was pressed into a circular groove in the bedplate, at the bottom of the bell-jar ; the angle iron was bolted down by 24 bolts exerting a force of 50 tons on the fuse wire gasket, to which atmospheric pressure on exhausting added another 10 tons.

The mirror surface must be absolutely clean for a satisfactory coat. The most satisfactory way of securing the requisite cleanliness with a large mirror was by removing foreign material with an electric discharge from a central electrode. With the arrangements used for the Crossley mirror a very uniform thickness (about $\frac{1}{1} \delta \mu$ ) of coat was obtained.

Dr. Strong finds that it is possible to coat speculum metal gratings with aluminium; the reflectivity is increased about 50 per cent for visible light and by an even greater extent in the ultra-violet. The higher orders of spectrum also become relatively brighter. The definition was found not to be impaired by the coating. The coat can be dissolved by caustic potash, which does not attack the speculum metal. This application of the process should prove of value in the laboratory as well as in astronomical spectroscopy.

It is hoped that apparatus for aluminising telescope mirrors up to a size of 36 inches diameter will be available in Great Britain before long. Some experimental work has been in progress, and a piece of plate-glass coated with aluminium was on view at the meeting of the Royal Astronomical Society on March 9.

\section{The Lyochromes: a New Group of Animal Pigments}

\section{By Phictpr Elunnger and Walter Koschara, Düsseldorf}

$\mathrm{I}^{\mathrm{N}}$ the observations of living animal organs by the 'intravital microscope'1 it was noticed that cells of some organs of the animal not previously treated with fluorescent dyestuffs contained substances which were excited by ultra-violet light to give a characteristic yellow-green fluorescence. Such substances were chiefly found in the liver cells and in the epithelial cells of the first convoluted tubules of the kidney of all the animals examined, including horses, oxen, dogs, cats, rabbits, guinea pigs, rats, mice, frogs, etc. In the liver two other groups of cells could be found which were fluorescent, but far less strongly; the Kuppfer star-cells, shining with a dull orangeyellow fluorescence, and single cells, showing a reddish fluorescence, near to the blood vessels. The two groups last named have no connexion with the pigments with which this article deals. The intensity of the fluorescence of the epithelial cells of the kidney, and also of the liver cells, appeared to be diminished when the animals (rats) were fed on a diet free from nitrogen, and to be augmented after the administration of urea. The suggestion arose that these pigments might have some connexion with the formation or the excretion of urea, because they were found in great quantity just at the sites of the intensive formation and excretion, and therefore of great concentration, of urea. Since it appeared likely that these pigments had great physiological importance, we tried to isolate and identify them.

Animal pigments have been for a long time an object of interest to physiologists and chemists. Especially by the researches of chemists, knowledge of them has been greatly increased during recent decades. The animal pigments hitherto known are nearly all soluble in neutral organic solvents under suitable conditions, and this property was highly important for their isolation and recognition. Only a few of the animal pigments previously known are strongly fluorescent, and their fluorescence differs in colour from that of the pigments now found in the kidney and the liver.

The first attempts at extraction showed that our pigments were completely insoluble in the usual indifferent solvents, such as ether, chloroform, benzol, ligroin, and that, on the other hand, they were soluble in water. As a guide in our attempts at isolation we used the characteristic yellow-green fluorescence, which clearly revealed even extremely small quantities, and which was further characterised by the fact that it was to a high degree dependent on the $p \mathrm{H}$, being changed reversibly to a violet fluorescence by both acids 